\title{
How does mechanism of biosorption determine the differences between the initial and equilibrium adsorption states?
}

\author{
Władysław Rudzinski • Wojciech Plazinski
}

Received: 9 April 2010 / Accepted: 4 May 2010 / Published online: 25 June 2010

(C) The Author(s) 2010. This article is published with open access at Springerlink.com

\begin{abstract}
The analysis of various models assumed to represent the influence of $\mathrm{pH}$ on heavy metals biosorption equilibrium is presented. It shows that all of them lead to the same mathematical expressions (e.g. the Langmuir or the Flory adsorption isotherm equations) when the $\mathrm{pH}$ effects are neglected. Even if considering the $\mathrm{pH}$ effects, some of them (competitive adsorption and ion-exchange models, for instance) still lead to analogical expressions for sorption isotherm equations. The accepted mechanism of biosorption may, however, influence strongly the differences between the initial and equilibrium states of biosorption system.
\end{abstract}

Keywords Biosorption $\cdot$ Ion exchange $\cdot$ Competitive adsorption $\cdot$ Non-competitive adsorption $\cdot \mathrm{pH}$

$\begin{array}{ll}\text { Abbreviations } \\ \mathrm{M} & \text { metal ion } \\ \mathrm{X} & \text { binding site } \\ K_{a} & \text { deprotonation constant } \\ N_{s} & \text { concentration of binding sites } \\ q & \text { sorbed amount of metal } \\ n, \pi & \text { constants related to the metal binding reaction } \\ m & \text { mass of sorbent } \\ \Delta & \text { difference between quantities in (12)-(14) } \\ x & \text { dimensionless concentration of metal ions in the } \\ & \text { bulk phase } \\ \kappa_{\mathrm{A}}-\kappa_{\mathrm{E}} & \text { constants depending on the considered model }\end{array}$

W. Rudzinski · W. Plazinski ( $₫)$

Institute of Catalysis and Surface Chemistry, Polish Academy of Sciences, ul. Niezapominajek 8, 30-239 Cracow, Poland e-mail: wojtek_plazinski@o2.pl

W. Rudzinski

Department of Theoretical Chemistry, Faculty of Chemistry,

UMCS, pl. M. Curie-Sklodowskiej 3, 20-031 Lublin, Poland

\section{Introduction}

Biosorption is a promising and innovative technology using inactive and dead biomasses to remove heavy metals from aqueous solutions. The experimental measurements revealed that $\mathrm{pH}$ is one of the most important technical factors influencing the biosorption efficiency (Veglió and Beolchini 1997; Volesky 2001; Volesky and Holan 1995). In many cases the starting point for quantitative and qualitative description of $\mathrm{pH}$-dependent equilibria in biosorption systems is to formulate some simple equations corresponding to physical and chemical processes occurring on the biosorbent surface. These equations usually originate from the mass-action law and are defined by expressing thermodynamic equilibrium constants through the appropriate concentrations of ions in both the bulk solution and the adsorbed phase. Such a procedure was accepted in many cases to develop more advanced models and led to successful description of the data measured in many important metal/biosorbent systems (Fourest and Volesky 1996; Schiewer and Volesky 1997; Yun et al. 2001)

The correct choice of elementary reactions, presumably responsible for binding metal ions, is obviously essential for further success in modeling of the whole process. Moreover, as it has recently been proved, even the method of formulation of mathematical dependencies can significantly influence the features of developed model. Plazinski and Rudzinski have shown that the 1:2 stoichiometric assumption should correspond to the $\mathrm{MX}_{2}$-type surface complexes ( $\mathrm{M}$ is the metal ion and $\mathrm{X}$ is the binding site) but not to the $\mathrm{M}_{1 / 2} \mathrm{X}$ complexes, which are frequently assumed to exist (Plazinski and Rudzinski 2010). The latter approach does not take into account the correlation between sites binding a single metal ion and can be used only as an approximate method, giving correct results for relatively high metal 
concentrations in the bulk solution. In view of these results studying the basic elementary reactions used for description of equilibria in the binary (i.e. containing metal ions and protons) sorption systems is justified.

The main aim of this paper is to present and compare the most popular models applied for modeling the dependence of metal ions uptake on $\mathrm{pH}$ of the solution in biosorption systems. Biosorbents are usually extremely diverse materials and, due to their complexity, are hard to model in detail. Therefore, all models presented here should be treated as "starting points" for building some more advanced description of biosorption phenomena.

\section{The description of models}

As the effect of acid-base properties of biosorbent is included in most models presented here, let us define the $K_{a}$ parameter as the constant of deprotonation of binding sites. It can be expressed by the following equation:

$K_{a}=\frac{\left[\mathrm{X}^{-}\right] \cdot 10^{-\mathrm{pH}}}{[\mathrm{HX}]}$.

in which $\left[\mathrm{X}^{-}\right]$is the equilibrium concentration of free binding sites, whereas $[\mathrm{HX}]$ is the concentration of sites occupied by protons. Both these values are expressed in $[\mathrm{mol} / \mathrm{g}]$. For the sake of simplicity, the assumption that there exists only one kind of monovalent binding sites was accepted. The total concentration of binding sites will be denoted as $N_{s}$. Depending on the accepted model, it can be com-

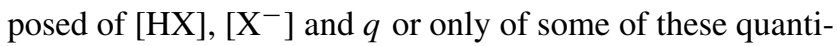
ties ( $q$ is the amount of metal on the surface in $[\mathrm{mol} / \mathrm{g}]$ ). The analytical solutions for $q(C, \mathrm{pH})$ are possible in the case of all models presented here, where $C$ is the metal ion concentration in the bulk solution. Their condition is that the number of sites involved in binding a single metal ion $(n)$ must be a small integer, which corresponds to the majority of physical situations. However, it is impossible to obtain the solution for $q$, maintaining $n$ as an indeterminate parameter, i.e. $q(C, \mathrm{pH}, n)$. Thus, considering the number of possible solutions for all $n$ 's, we decided to present the equilibrium sorption isotherm equations in the form derived directly from the mass action law.

Furthermore, defining $K_{a}$ having only one value does not reflect the surface heterogeneity which is characteristic of many biosorption systems. On the other hand, the studies considering the heterogeneity effects in the related systems are already known in the literature (Plazinski and Rudzinski 2009b; Fourest and Volesky 1996). In the present study both $K_{a}$ and equilibrium constants appearing in the isotherm equations of metal adsorption should be viewed in terms proposed by Garces et al. (2002) (i.e. as the so-called "average equilibrium functions") or as the parameters describing only some part of the system under consideration.
2.1 Two-component competitive adsorption model (Model A)

The model presented here originates from the classical approach proposed for the description of adsorption in binary systems. Its main assumptions can be summarized as follows:

- uptake of both metal ions and protons is possible only through adsorption onto free binding sites (in the case of metal the multi-site-occupancy adsorption is allowed);

- influence of $\mathrm{pH}$ is seen as the effect of competition for free sites between metal ions and protons.

Two reactions corresponding to this model can be represented as follows:

$\mathrm{M}^{n+}+n \mathrm{X}^{-} \longleftrightarrow \mathrm{MX}_{n}$

$\mathrm{H}^{+}+\mathrm{X}^{-} \longleftrightarrow \mathrm{HX}$.

The equilibrium sorption isotherm equation has the following form:

$\kappa_{\mathrm{A}}=\frac{q}{C} \frac{\left(10^{-\mathrm{pH}}+K_{a}\right)^{n}}{K_{a}^{n}\left(N_{s}-n q\right)^{n}}$

where $\kappa_{\mathrm{A}}$ is the equilibrium constant for reaction (2). Its dimension is $\left[\mathrm{L} \cdot \mathrm{g}^{n-1} \cdot \mathrm{mol}^{-n}\right]$. When $n=1$, (4) reduces to the so-called multicomponent Langmuir (MarkhamBenton) isotherm equation. Another important observation is that (4) is mathematically equivalent to (i) the Langmuir isotherm equation for $n=1$; (ii) Flory's isotherm equation for $n \neq 1$. Then, some coefficients appearing in these two expressions become $\mathrm{pH}$-dependent. Thus, when neglecting the description of $\mathrm{pH}$-related effects, the Langmuir (or Flory's) equation is able to correlate experimental data equally well as Model A considered here.

This model (and its modification) is widely used for description of $\mathrm{pH}$ influence on the biosorption equilibrium. However, reactions (2) and (3) do not explicitly take into account the process of ion exchange which can be considered as crucial for many important biosorption systems (Crist et al. 1994; Veglió and Beolchini 1997).

\subsection{Non-competitive adsorption model (Model B)}

The above model was proposed on the empirical basis to explain some features of experimental systems (Veglió et al. 2002). Further, it was generalized by incorporating the surface energetic heterogeneity of typical biosorbents (Plazinski and Rudzinski 2009a). Here, the form developed for the model of a homogeneous surface is considered. The most important features of Model B are listed below: 
- as in the case of Model A, binding of both metal ions and protons occurs through adsorption onto free sites (multisite-occupancy adsorption is possible for metal ions);

- adsorption of protons is not practically affected by the presence of metal ions whereas metal ions can be adsorbed on the binding sites which are not occupied by protons;

- the latter assumption leads to the conclusion that the influence of $\mathrm{pH}$ on the sorption isotherm is restricted to the regulation of the number of binding sites available for metal ions.

This model can be also represented by the reaction equations (2) and (3). Also, the definition of the $\kappa_{\mathrm{B}}$ constant (see (5)) and its dimension are identical to those valid for $\kappa_{\mathrm{A}}$. The difference lies in the equilibrium isotherm equation (5) representing Model B:

$\kappa_{\mathrm{B}}=\frac{q}{C}\left(\frac{N_{s} \cdot 10^{-\mathrm{pH}}}{K_{a}+10^{-\mathrm{pH}}}-n q\right)^{-n}$.

For $n=1$, (5) reduces to the form already known in the literature (Veglió et al. 2002).

One of the main advantages of Model B is the fact that it is able to correctly predict the behaviour of many real biosorption systems, observed experimentally for high values of $C$. Namely, when considering the set of a few sorption isotherms, measured for different (fixed) $\mathrm{pH}$ values (i.e. $\left.q\left(C, \mathrm{pH}_{1}\right), q\left(C, \mathrm{pH}_{2}\right), q\left(C, \mathrm{pH}_{3}\right), \ldots\right)$ one can observe that the maximum uptake of metal depends only on a given $\mathrm{pH}$ value and not on the increasing value of $C$. This situation can be mathematically illustrated by the following expression:

$$
\begin{aligned}
\lim _{C \rightarrow \infty} q\left(C, \mathrm{pH}_{1}\right) & \neq \lim _{C \rightarrow \infty} q\left(C, \mathrm{pH}_{2}\right) \\
& \neq \lim _{C \rightarrow \infty} q\left(C, \mathrm{pH}_{3}\right) \neq N_{s} .
\end{aligned}
$$

More details related to this model can be found in one of our previous papers (Plazinski and Rudzinski 2009a).

\subsection{Ion exchange model (Model C)}

The principles of the ion exchange model are generally wellknown in the literature and it is widely applied to analyze experimental biosorption systems. Its generalizations, including the effects of chemical heterogeneity, can be found elsewhere (Plazinski and Rudzinski 2009b). Here, the simplest case is taken into account. The general assumptions inherent in Model $\mathrm{C}$ are following:

- binding of metal ions is the ion-exchange process in which metal ion displaces proton (or protons, if $n>1$ ).

- there are no free binding sites on the surface; all of them are occupied by protons or by metal ions;
- influence of $\mathrm{pH}$ is expressed by the general law of mass action.

For Model C, the reaction for the binding of metal is:

$\mathrm{M}^{n+}+n \mathrm{HX} \longleftrightarrow \mathrm{MX}_{n}+n \mathrm{H}^{+}$

and the corresponding sorption isotherm is represented by (8):

$\kappa_{\mathrm{C}}=\frac{q}{C} \frac{10^{-n \mathrm{pH}}}{\left(N_{s}-n q\right)^{n}}$

where $\kappa_{\mathrm{C}}$ is the equilibrium constant for exchange reaction (7) having a dimension $\left[\mathrm{g}^{n-1} \cdot \mathrm{L}^{n-1}\right]$.

Model C can be applied for description of the systems in which the ion exchange process has been experimentally confirmed by monitoring the systematic release of protons in the course of metal ion sorption. In particular, this effect concerns many algae-based systems (Crist et al. 1994; Davis et al. 2003). One of the main weaknesses of this model, however, is that it neglects the presence of a number of free binding sites (i.e. it assumes that the biosorbent is fully protonated). One can observe that (8) is $K_{a}$ independent, for instance. The extension of this model is given in the next section and denoted as Model D.

As in the case of Model A, the equilibrium sorption isotherm equation (8) is mathematically equivalent to the Langmuir isotherm equation or to the Flory isotherm equation, depending on the value of $n$. Also in this case the parameters of these two expressions should be treated as functions of $\mathrm{pH}$. This observation has been previously made for $n=1$ (see Crist et al. 1994 for more details).

\subsection{Ion exchange model including the acid-base properties of the biosorbent (Model D)}

This model is very similar to Model $\mathrm{C}$ but it is further extended by taking into account the acid-base properties of the biosorbent surface. This assumption can be incorporated into mathematical models by accepting some degree of deprotonation of binding sites characterized by reaction (3) and constant (1). Some basic assumptions related to Model D are following:

- analogically to Model $\mathrm{C}$, the binding of metal ions is driven only by the ion-exchange process in which metal ion displaces proton(s).

- binding sites may be free or occupied by protons or by metal ions;

- the effect of $\mathrm{pH}$ is expressed by both the law of mass action related to both free binding sites and those occupied by metal ions. The acid-base properties of biosorbent surface are characterized by the value of $K_{a}$. 
The reactions corresponding to this model are represented by (3) and (7), whereas the equilibrium state can be described by:

$\kappa_{\mathrm{D}}=\frac{q}{C} \frac{\left(10^{-\mathrm{pH}}+K_{a}\right)^{n}}{\left(N_{s}-n q\right)^{n}}$.

As one can see, contrary to (8), the above formula depends on the $K_{a}$ parameter. The definition and dimension of the $\kappa_{\mathrm{D}}$ constant are identical to those introduced for $\kappa_{\mathrm{C}}$.

\subsection{Model including both the ion exchange and adsorption processes (Model E)}

This model seems to be the most general one of the presented ones, as is takes into account both the adsorption on the free sites and ion exchange processes. Let us write the following reaction of binding a metal ion:

$\mathrm{M}^{n+}+\pi \mathrm{HX}+(n-\pi) \mathrm{X}^{-} \longleftrightarrow \mathrm{MX}_{n}+\pi \mathrm{H}^{+}$

for which the sorption isotherm equation (being actually the equilibrium constant of reaction (10)) takes the form:

$\kappa_{\mathrm{E}}=\frac{q}{C} \frac{\left(10^{-\mathrm{pH}}+K_{a}\right)^{n}}{\left(N_{s}-n q\right)^{n} K_{a}^{n-\pi}}$.

The dimension of $\kappa_{\mathrm{E}}$ is $\left[\mathrm{L}^{1-\pi} \cdot \mathrm{g}^{n-1} \cdot \mathrm{mol}^{\pi-n}\right]$. As one can see, (11) differs from both equations (4) and (9) only very slightly, by the presence of the $K_{a}^{n-\pi}$ constant in the denominator at the 1.-h.-s. of (11). It means that all these three expressions are able equally accurately predict the influence of $\mathrm{pH}$ on the biosorption equilibrium, however, the values of some parameters obtained using them will probably be different. This observation is mainly related to the $\kappa_{\mathrm{A}}, \kappa_{\mathrm{E}}$ and $\kappa_{\mathrm{D}}$ constants. As the relation $\kappa_{\mathrm{A}} K_{a}^{n}=\kappa_{\mathrm{D}}=\kappa_{\mathrm{E}} K_{a}^{n-\pi}$ is valid, applying (9) or (4) instead of (11) may lead to "apparent" $\kappa_{\mathrm{D}}$ or $\kappa_{\mathrm{A}}$ values but the quality of the data description (expressed in terms of the error function) remains unchanged.

It is worth noting that incorporating the adsorption process into Model D requires introducing only one additional parameter $(\pi)$ for which the lowest and highest values are known. When $\pi=n,(10)$ and (11) reduce to (7) and (9), respectively, i.e. the biosorption process is ion-exchangedriven, whereas when $\pi=0$, one has to deal with purely adsorption process, which can be modeled by using (2)-(4).

\section{Some observations}

Models A, D and E can be considered as pH-dependent editions of the Langmuir isotherm equation (for $n=1$ ) or, in the more general case, of the Flory isotherm equation (for $n \neq 1$ ) whereas Models B and C correspond to the Langmuir equation (for $n=1$ ). Therefore, both these equations are equally suitable to correlate the experimental $(q, C)$ data as the models presented in the previous section; they are not able to account for the $\mathrm{pH}$-related effects, however. A similar observation has already been made concerning Model A (Crist et al. 1994; Plazinski and Rudzinski 2009a). Thus, the fact of good correlation of the data by these mathematical expressions should not lead to drawing any reliable conclusions about mechanisms governing the biosorption processes. The additional complication is connected with the fact that both the multidentate binding mode and surface heterogeneity effects may cause very similar changes in the behaviour of the measured $q(C)$ sorption isotherm (Plazinski and Rudzinski 2009b).

In spite of the most general character of reaction (10), it should be considered only as a crude approximation of real processes occurring at the surface. Two main reasons for that are as follows. (i) Reaction (10) expresses binding of single metal ion as "shared" between adsorption and ion exchange processes. In fact, both these processes may occur in parallel only in macroscopic scale and (10) is merely a simplified mathematical notation of this fact. (ii) When significant changes of surface coverage by protons and metal ions are observed in the course of metal binding, the "share" of a given process, represented by the value of $\pi$ can be considered as time-dependent. In other words, the number of protons replaced by single metal ion may depend on the probability of finding a binding site occupied by proton. In this case the value of $\pi$ is time-dependent and results from the equilibrium and kinetic properties of the system.

The analogical forms of mathematical expressions corresponding to Models A, D and E can not be surprising when considering the thermodynamic description of biosorption systems representing these models. In this case one can distinguish two phases (the adsorbed phase and the bulk solution) containing two different sorbates (protons and metal ions). The state of equilibrium requires the equality of chemical potentials of a given species in both considered phases. When considering only the phase located on the surface, the chemical potentials of the adsorbed ions depend only on their surface coverages and on some properties of the system. The direct consequence of this fact is that the mathematical form of expressions describing the equilibrium may be independent of the type of process responsible for binding metal ions. In the case of Models A, D and E one has to note that if two different processes (adsorption and ion exchange) lead to the same values of surface coverages by protons and metal ions, then determination of the kind of surface reaction has no influence on the general form of sorption isotherm equations. It may, however, influence the theoretical interpretation of some constants (here: $\kappa_{\mathrm{A}}, \kappa_{\mathrm{D}}$ 
and $\left.\kappa_{\mathrm{E}}\right)$. Moreover, the situation can be much more complicated in such cases as adsorption on the energetically heterogeneous surface (considered in Plazinski and Rudzinski $2009 \mathrm{~b}$ in the context of ion-exchange process).

In the face of the above mentioned observations, the differentiation between ion-exchange (Model D), competitive adsorption (Model A) and intermediate (Model E) processes does not seem to have significant importance as long as the original conditions (e.g. the surface homogeneity) are fulfilled to an acceptable level. This statement is generally true when considering only the equilibrium properties of the system. Significant differences can appear in the case of analysis including especially the kinetic features or, for instance, the comparison of the equilibrium (final) state of the biosorption system with the initial one. The former issue is the matter of the forthcoming studies; the latter is discussed in the present paper.

Simple model calculations were performed on the basis of Models A, D and E in order to compare the difference between the initial and the final $\mathrm{pH}$ values for the systems corresponding to the same $q(C, \mathrm{pH})$ equilibrium sorption isotherm. Models B and $\mathrm{C}$ were not included as the former does not assume any changes of $\mathrm{pH}$ and the latter is oversimplified as it does not allow for the existence of free binding sites. Model E was considered as being the transitional one between Models A and D.

The expressions accounting for mass-balance relations for both metal ions and protons and corresponding to Models A, D and E can be written as (12), (13) and (14), respectively:

$$
\begin{aligned}
\Delta\left(10^{-\mathrm{pH}}\right) V & =-m \Delta[\mathrm{HX}], \\
\Delta\left(10^{-\mathrm{pH}}\right) V & =m n \Delta q-m \Delta[\mathrm{HX}], \\
\Delta\left(10^{-\mathrm{pH}}\right) V & =m \pi \Delta q-m \Delta[\mathrm{HX}] .
\end{aligned}
$$

$\Delta$ denotes the difference between the final and initial values of a given quantity, $m$ is the mass of sorbent and $V$ is the volume of bulk solution. The initial state is represented by the partially protonated biosorbent being in equilibrium with a bulk solution of $\mathrm{pH}_{i n}$; no metal ions are initially adsorbed on the biosorbent surface. The equilibrium is described by the expression analogical to (1). The relation $N_{s}=[\mathrm{HX}]+\left[\mathrm{X}^{-}\right]=n q$ is common for all the studied models. As our attempts were directed at comparison of the systems represented by the same mathematical form of equilibrium sorption isotherms ((4), (9) and (11)), the unified, dimensionless scale of metal ion concentration was used and defined as: $x=\kappa_{\mathrm{A}} K_{a}^{n} C=\kappa_{\mathrm{D}} C=\kappa_{\mathrm{E}} K_{a}^{n-\pi} C$.

Figure 1 shows the results concerning the influence of the accepted model, expressed by the value of $\pi$, whereas Fig. 2 presents the effects related to different solid/solution ratios. Main findings can be summarized as follows:

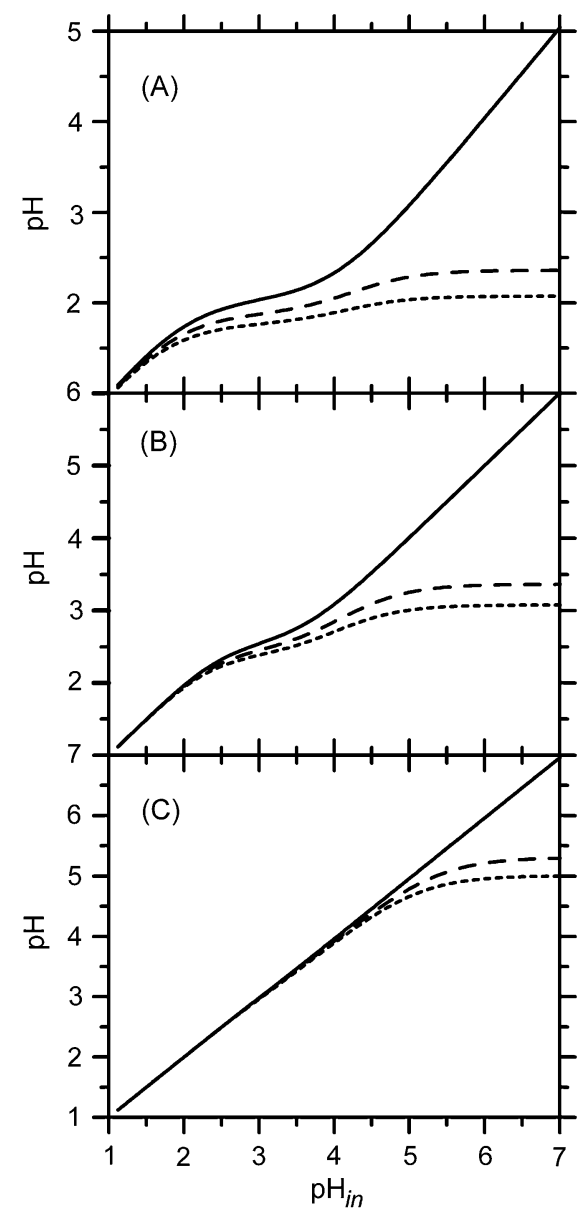

Fig. 1 The dependence of the equilibrium on the initial $\mathrm{pH}$ value for the three different models of biosorption: Model A ( $\square)$, Model D (- - - ), Model $\mathrm{E}$ for $\pi=0.5\left(--\mathrm{-}^{-}\right)$and the three different values of the $x$ parameter: $x=10^{-1}(\operatorname{panel}(\mathbf{A})), x=10^{-3}(\operatorname{panel}(\mathbf{B})), x=10^{-5}$ (panel (C)). The values of other parameters: $n=1, N_{s}=0.002 \mathrm{~mol} / \mathrm{g}$, $m / V=5 \mathrm{~g} / \mathrm{L}$

- The dependence of $\mathrm{pH}$ on $\mathrm{pH}_{\text {in }}$ is of different character in the case of each considered model. The ion-exchange model (Model D) predicts the most significant changes of $\mathrm{pH}$ as the number of released protons per single metal ion is the highest. On the other hand, the release of protons in the case of the competitive adsorption model (Model A) is the result of disturbance in equilibrium between protons in the bulk solution and those in the adsorbed phase; the disturbance is caused by changes in the composition of that phase (i.e. by binding metal ions). Model E predicts in-between situations, depending on the value of $\pi$.

- As expected, differences between prediction of all three models tend to disappear at low uptakes of metal. It is understandable, as very small uptake of metal ions is not able to cause any significant change in the surface protonation degree (compare Figs. 1(A), (B) and (C), representing the decreasing value of metal ion equilibrium concentrations). 


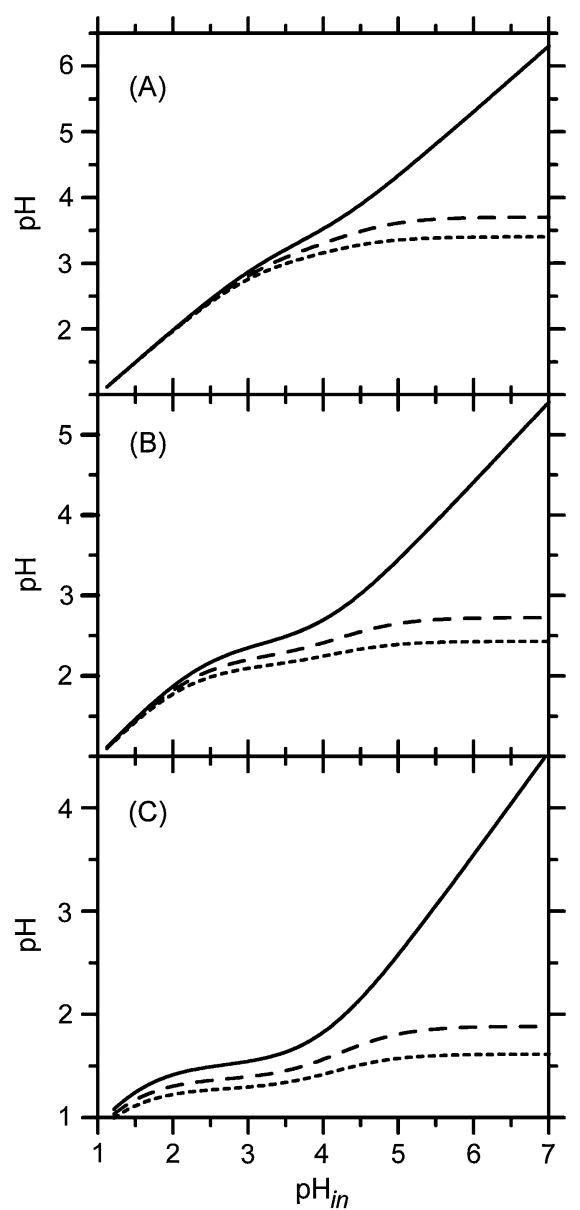

Fig. 2 The dependence of the equilibrium on the initial $\mathrm{pH}$ value for the three different models of biosorption: Model A ( $\square$ ), Model D (- - - ), Model E for $\pi=0.5$ (- - - -) and the three different values of the solid solution ratio: $m / V=0.2 \mathrm{~g} / \mathrm{L}$ (panel (A)), $m / V=2 \mathrm{~g} / \mathrm{L}$ (panel $(\mathbf{B})), m / V=20 \mathrm{~g} / \mathrm{L}$ (panel $(\mathbf{C}))$. The values of other parameters: $n=1, N_{s}=0.002 \mathrm{~mol} / \mathrm{g}, x=10^{-1}$

- Apart from the accepted model, the physical features of the considered system are at least equally important. Figure 2 presents the behaviour of the systems represented by different values of the solid/solution ratio. The differences between models (as well as those between the initial and final pHs) become more significant at high $m / V$ values.

- Observations analogical to those mentioned above can be also made for the values of $n$ different from unity (data not shown).

\section{Concluding remarks}

The simple comparative analysis of models presented in Sect. 2 revealed that both Model A (corresponding to the competitive adsorption mechanism) and Model D (based on accepting the ion-exchange reaction) lead to the same mathematical expressions describing the state of equilibrium in the binary sorption system containing protons and metal ions. However, the consequences of accepting a given model may have appreciable influence on: (i) the theoretical interpretation of some parameters, especially the equilibrium constants; (ii) the prediction of equilibrium state on the basis of the known initial conditions. The latter issue has been illustrated by the comparison of dependencies of $\mathrm{pH}$ on $\mathrm{pH}_{\text {in }}$ for the selected models. Thus, the analysis of final (equilibrium) and initial features of the system may serve as a simple tool suggesting the mechanism of binding metal ions. Furthermore, accepting the elementary reaction (or the set of such reactions) influences strongly the attributes of developed models. It was shown here on the basis of the models differentiating between the ion-exchange/adsorption processes, whereas in our previous papers a similar conclusion has been drawn for two different definitions of surface complexes corresponding to the same binding stoichiometry (Plazinski and Rudzinski 2010). As the biosorption processes are usually very complicated, all models presented here are nearly first approximation which may be used to build some more advanced descriptions. Further theoretical studies focused on elementary processes occurring during biosorption are needed.

Acknowledgements One of the authors (W.P.) acknowledges the financial support of the Foundation for Polish Science (START program, 2009 and 2010). This work was supported by Polish Ministry of Science and Higher Education (contract financed in 2010-2012) under the Project No. N N204 271238.

Open Access This article is distributed under the terms of the Creative Commons Attribution Noncommercial License which permits any noncommercial use, distribution, and reproduction in any medium, provided the original author(s) and source are credited.

\section{References}

Crist, R.H., Martin, J.R., Carr, D., Watson, J.R., Clarke, H.J., Crist, D.R.: Interaction of metals and protons with algae. 4. Ion exchange vs adsorption models and a reassessment of Scatchard plots; ion-exchange rates and equilibria compared with calcium alginate. Environ. Sci. Technol. 28, 1859-1866 (1994)

Davis, T.A., Volesky, B., Mucci, A.: A review of the biochemistry of heavy metal biosorption by brown algae. Water Res. 37, 43114330 (2003)

Fourest, E., Volesky, B.: Contribution of sulfonate groups and alginate to heavy metal biosorption by the dry biomass of Sargassum fluitans. Environ. Sci. Technol. 30, 277-282 (1996)

Garces, J.L., Mas, F., Cecılia, J., Companys, E., Galceran, J., Salvador, J., Puy, J.: Complexation isotherms in metal speciation studies at trace concentration levels. Voltammetric techniques in environmental samples. Phys. Chem. Chem. Phys. 4, 3764-3773 (2002)

Plazinski, W., Rudzinski, W.: Modeling the effect of $\mathrm{pH}$ on kinetics of heavy metal ion biosorption. A theoretical approach based on the statistical rate theory. Langmuir 25, 298-304 (2009a)

Plazinski, W., Rudzinski, W.: Modeling the effect of surface heterogeneity in equilibrium of heavy metal ion biosorption by using the ion exchange model. Environ. Sci. Technol. 43, 7465-7471 (2009b) 
Plazinski, W., Rudzinski, W.: Binding stoichiometry in sorption of divalent metal ions: a theoretical analysis based on the ion-exchange model. J. Colloid Interface Sci. 344, 165-170 (2010)

Schiewer, S., Volesky, B.: Ionic strength and electrostatic effects in biosorption of divalent metal ions and protons. Environ. Sci. Technol. 31, 2478-2485 (1997)

Veglió, F., Beolchini, F.: Removal of metals by biosorption: A review. Hydrometallurgy 44, 301-316 (1997)

Veglió, F., Esposito, A., Reverberi, A.P.: Copper adsorption on calcium alginate beads: equilibrium pH-related models. Hydrometallurgy 65, 43-57 (2002)
Volesky, B.: Detoxification of metal-bearing effluents: Biosorption for the next century. Hydrometallurgy 59, 203-216 (2001)

Volesky, B., Holan, Z.R.: Biosorption of heavy metals. Biotechnol. Prog. 11, 235-250 (1995)

Yun, Y.-S., Park, D., Park, J.M., Volesky, B.: Biosorption of trivalent chromium on the brown seaweed biomass. Environ. Sci. Technol. 35, 4353-4358 (2001) 